\title{
BIOBASED INTELLIGENT PACKAGING APPLICATION
}

\author{
Aslı Beyler Çiğil (D) \\ Amasya University Technical Sciences Vocational, Department of Chemistry and Chemical \\ Process Technology School, Amasya, Turkey
}

\begin{abstract}
Changes in consumer preferences in order to reach safe food have led to innovations in packaging technologies. Intelligent and active packaging is a constantly developed packaging technology that plans to offer safer and higher quality products. Active packaging refers to the inclusion of additives in the package in order to maintain and / or extend shelf life and product quality. Intelligent systems, on the other hand, are systems that monitor the status of packaged food during this entire period to provide information about the quality of the packaged during storage and transportation. The aim of this study is to produce a completely natural intelligent packaging material using rosehip extract and biopolymer, which is a substance that naturally changes color with $\mathrm{pH}$. In this study, cellulose acetate butyrate biobased films containing different rates $(1,2.5,5,10 \mathrm{wt} \%)$ of rosehip extract were produced by solvent casting method. The chemical structure the rosehip containing biobased film and blank biobased film were characterized by ATR-FTIR. The transparency of prepared five different films were determined by UV spectroscopy. The color characteristic of blank and rosehip containing films measured with spectrophotometer. Surface energy of all films and contact angles were determined with goniometer. Biobased films were printed and printability parameters such as color, gloss, contact angle, surface tension were examined. It is concluded that blank biobased film is colorless, transparent and all biobased films have good printability. It was determined that the amount of rosehip extract increased the color change visibly. The biobased films obtained are pink in acidic medium and yellow-green in alkaline medium. The results prove that biobased film produced with rosehip extract and cellulose acetate butyrate can be used in intelligent packaging applications.
\end{abstract}

Keywords: intelligent packaging, biobased films, rosehip, indicator

\section{INTRODUCTION}

Packaged foods such as takeaway and fast food have become an important component of daily life with the intensification of business life. The safety of all food used is the most important element of public health in the society (Ghasemi-Varnamkhasti et al, 2018). Perishable foods seriously adversely affect people's health, but determining the freshness of some foods is very difficult (Idumah et al, 2020). Oldstyle food packaging methods are not intended to provide information about freshness, but only to protect food from external factors. Compared to the old-style packaging, smart packaging can provide information about the freshness of the packaged food, so the need for the development of new packaging has increased in order to preserve and determine the freshness of food. pH-sensitive packaging is defined as packaging on which a small and inexpensive pH-sensitive label is affixed to examine the freshness of the packaged food. When food spoilage begins, large amounts of inorganic or organic compounds are released and these compounds change the chemical component content, moisture and $\mathrm{pH}$ of the food. Developing a $\mathrm{pH}$ sensitive color-changing label or pattern on a food packaging can be a quick indicator of food freshness. They can be more time-saving, cheap, convenient and simple compared to the old-fashioned food packaging procedure (Liu et al, 2019; Zhang et al, 2019). Cellulose acetate butyrate (CAB) is a thermoplastic ester of cellulose, the most abundant natural polymer in nature. It contains $30 \%$ to $55 \%$ by weight of butyryl groups. It is a commercially important cellulose ester and has many attractive properties such as high transparency, improved resistance to moisture and ultraviolet light (UV), low viscosity, good interlayer adhesion and surface gloss. Cellulose acetate butyrate is compatbile with many different resin systems, including most acrylic, polyester, phenolic, ureas and isocyanates. $C A B$ has been an important component for coating formulations because it controls viscosity, improves leveling and flow, and reduces drying time (Saha et al, 2018; Laíse et al, 2020; Furtado et al, 2020). Cellulose acetate butyrate biocompatibility allows it to be used in applications such as antibacterial coatings, drug release system (Edgar, 2007; Sobral et al, 2008; Shokri and Adibkia, 2013) tissue engineering and effective support for enzymes. 
This study evaluated the influence of different concentrations rosehip extracts in CAB (cellulose acetate butyrate) polymeric films made by solvent casting, evaluating the printability, chemical, surface properties and color changes with $\mathrm{pH}$ for their potential application in intelligent packaging.

\section{METHODS}

Rosehip extraction: Rosehips (rosehip purchased at a local market) used in extraction experiments were dry fruits from Turkey. Whole fruits were ground to particles that were smaller than $2 \mathrm{~mm}$ and $5 \mathrm{~g}$ of dry and ground plant material were mixed with $50 \mathrm{~mL}$ of water. The colored mixture was separated from the solid particles by filtration. Two thirds of the solvent of the mixture was removed in the vacuum evaporator. The produced concentrated rosehip-water mixture in red-pink color was used directly in biobased films.

Preparation of biobased films: Biobased films were prepared by dissolving 10 grams of CAB (cellulose acetate butyrate) (CAB was obtained from Sigma Aldrich) in $100 \mathrm{~mL}$ of acetone and stirred until complete dissolution into the shaker. Then, rosehip extract were added to the CAB solution according to Table 1. The solutions were poured onto glass plate and dried under room temperature. Subsequently, biobased films was removed from the glass plate and stored in a vacuum desiccator before use. The average thickness of the biobased films were $10 \mu \mathrm{m}$.

Table 1: The biobased film formulations.

\begin{tabular}{|c|c|c|c|}
\hline Formulation & $\begin{array}{c}\text { CAB(cellulose } \\
\text { acetate butyrate } \\
\%\end{array}$ & Water \% & $\begin{array}{c}\text { Rosehip } \\
\text { extract \% }\end{array}$ \\
\hline C & 10 & 100 & 0 \\
\hline CR1 & 10 & 100 & 1 \\
\hline CR2.5 & 10 & 100 & 2.5 \\
\hline CR5 & 10 & 100 & 5 \\
\hline CR10 & 10 & 100 & 10 \\
\hline
\end{tabular}

Determination of printability: Base formulation film (C formulation) and rosehip added biobased films (CR1, CR2.5, CR5, CR10) were printed with IGT C1 test printing machine using equal amount of process magenta ink (DIN ISO 2846-1). Printing parameters; $0.2 \mathrm{~m} / \mathrm{s}$ printing speed and $300 \mathrm{~N}$ printing pressure. The polymeric ink film thickness of all biobased printed samples was measured as $5 \mu \mathrm{m}$.

Characterization: The color absorption character of rosehip dye with different $\mathrm{pH}$ was characterized with Shimadzu UV-Vis spectrophotometer 2450 (Kyoto Jap).

The transmission spectra of the biobased films were recorded in the wavelength range of 500-900 nm using a Shimadzu UV-vis spectrophotometer 2450 (Kyoto Jap).

Structural properties of all biobased films were measured with ATR-FTIR spectrum with Perkin Elmer Spectrum100 ATR-FTIR spectrophotometer.

The wettability properties of all biobased films were measured using the contact angle with the sessile water droplet method. The characteristics of surfaces were measured with contact angle (TAPPI T 458). Distilled water was used as standard wetting fluid in a Pocket Goniometer Model PG-X, (FIBRO Systems $A B$, Sweden), which was determined as a function of time.

Surface energies of all biobased films were measured on contact angle using ASTM D5946 standard test method.

The color measurements of the biobased films and prints on different films were made by CIE L*a*b* procedure using X-Rite eXact spectrophotometer according to ISO 12647-2:2013 standard. The measurement conditions of the spectrophotometer are measured as polarization filter with $0^{\circ} / 45^{\circ}$ geometry with 2 observer angle with D50 light source in the range of $400-700 \mathrm{~nm}$. The difference between the colors of the different prints were calculated according to formula 1 according to the CIE $\triangle E$ 2000 ISO 13655 standard.

$\Delta E_{00}=\sqrt{\left(\frac{\Delta L^{\prime}}{k_{L} S_{L}}\right)^{2}+\left(\frac{\Delta C^{\prime}}{k_{C} S_{C}}\right)^{2}+\left(\frac{\Delta H^{\prime}}{k_{H} S_{H}}\right)^{2}+R_{T} \frac{\Delta C^{\prime}}{k_{C} S_{C}} \frac{\Delta H^{\prime}}{k_{H} S_{H}}}$ 
The gloss measurements of biobased films were carried out with BYK Gardner GmbH micro gloss 750 geometry in accordance with ISO 8254-1:2009, and the gloss measurements of prints with BYK Gardner GmbH micro Tri-gloss 60 geometry in accordance with ISO 2813:2014.

\section{RESULTS AND DISCUSSION}

The functional groups in different biobased films, C, CR1, CR2.5, CR5 and CR10 formulations were determined by ATR-FTIR spectrum, and the spectra was given in Figure 1.

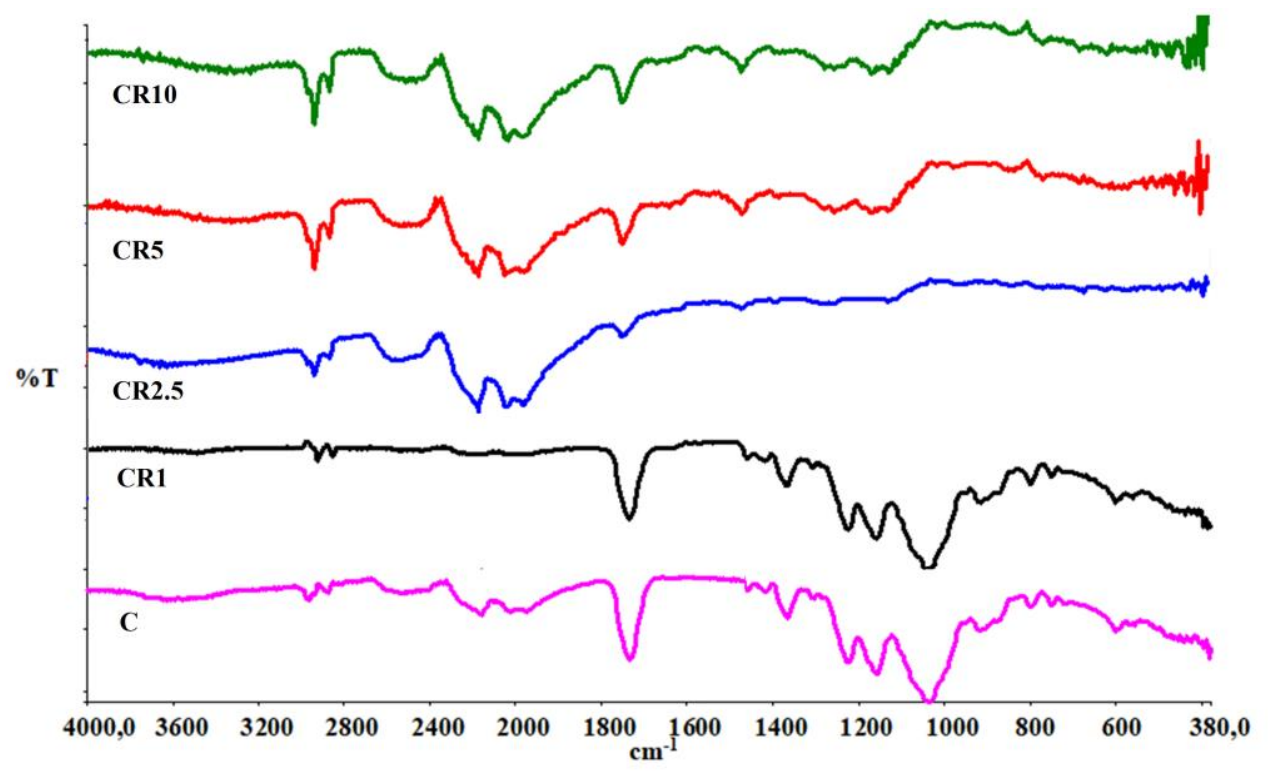

Figure 1: ATR-FTIR spectrums of all biobased films

The characteristic absorption bands that appeared at $2880 \mathrm{~cm}^{-1}$ in all the spectra, is due to the characteristic absorption peak of methylene associated with butyryl substituents in the cellulose acetate butyrate. The peak at approximately $1750 \mathrm{~cm}^{-1}$ represented ester carbonyl groups $(C=0)$ stretching vibrations in the cellulose acetate butyrate. The absorption peaks at 1050, 1238, 1376 and $3455 \mathrm{~cm}^{-1}$ were assigned $\mathrm{C}-\mathrm{O}$ stretching, $\mathrm{C}-\mathrm{O}-\mathrm{C}$ ether stretching vibration, $\mathrm{C}-\mathrm{H}$ bending and $\mathrm{O}-\mathrm{H}$ stretching vibration, respectively. The presence of metabolites such as phenolic acids, tannins, fatty acids, proanthocyanidins, flavonoids, pectin, fruit acids, sugars and carotenoids, such as malic acid, ascorbic acid and citric metabolites with antioxidant activity are known in fruits (rosehip) (Aimin Huang et al, 2020) Therefore, strong $\mathrm{O}-\mathrm{H}$ peaks are expected in CR1, CR2.5, CR5 and CR10 film spectra, but these peaks are not seen due to esterification between rosehip and CABs.

When Figure 2 is examined, it is determined that maximum absorbance of rosehip dyestuff in water shows a peak at $465 \mathrm{~nm}$. Absorption of the rosehip is compatible with magenta-red color. In addition, the obtained UV spectrum is in line with literature (Go and Song, 2019). In the UV spectrum, at acidic pHs, the wavelength value (also color) did not change, while the absorption value increased. So the color has become darker. In the UV spectrum, at alkaline pHs, the wavelength value (also color) changed and was determined to be $685 \mathrm{~nm}$. This value is compatible with the yellow-green color. The absorption value has decreased, is the color has become lighter. 


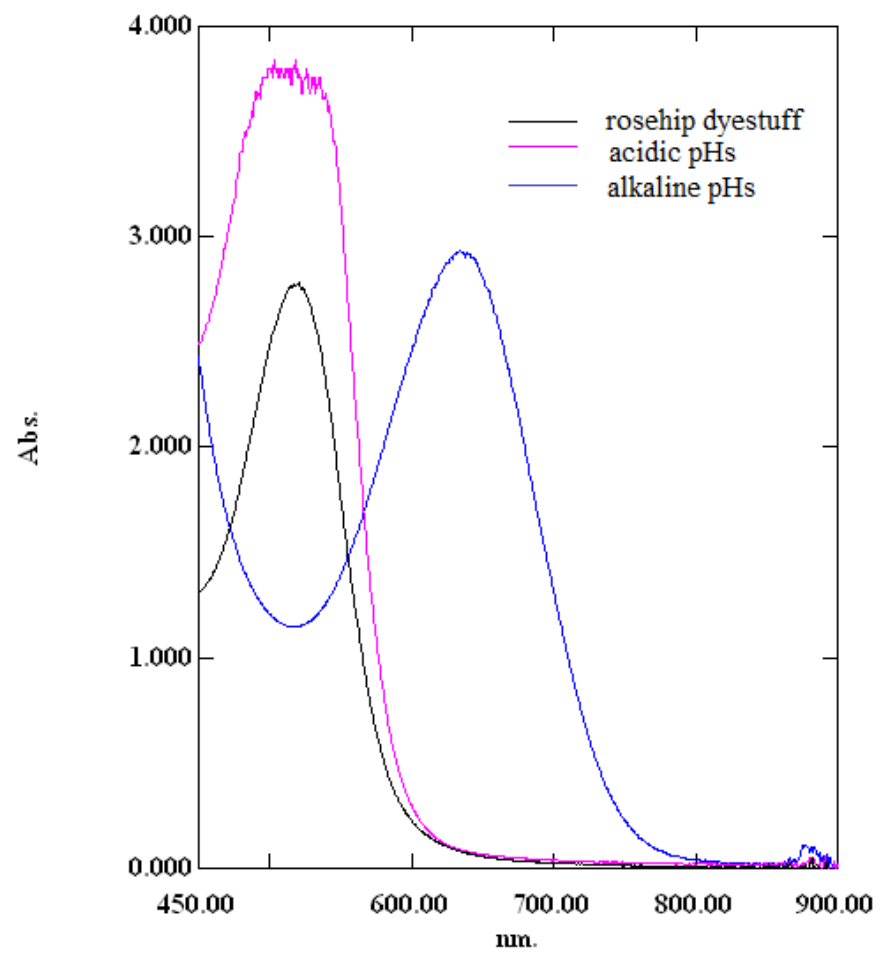

Figure 2: UV-vis absorption spectra of rosehip dyestuff

The optical transparencies of rosehip containing biobased films were characterized by UV-vis spectroscopy. Figure 3 shows the transmission spectra of rosehip containing films in the range between 500 and $900 \mathrm{~nm}$. Rosehip containing biobased films transmittance value is at $620 \mathrm{~nm}$. When the Figure 3 examined It was seen that transmittance of rosehip containing biobased macromolecular films decreased with increasing amount of rosehip amount. Since even the least amount of rosehip (CR1) causes an effective reduction in transparency, attention should be paid to its use in packaging applications that should be transparent. The obtained results are compatible with the literature (Çakmakçı and Güngör, 2013).

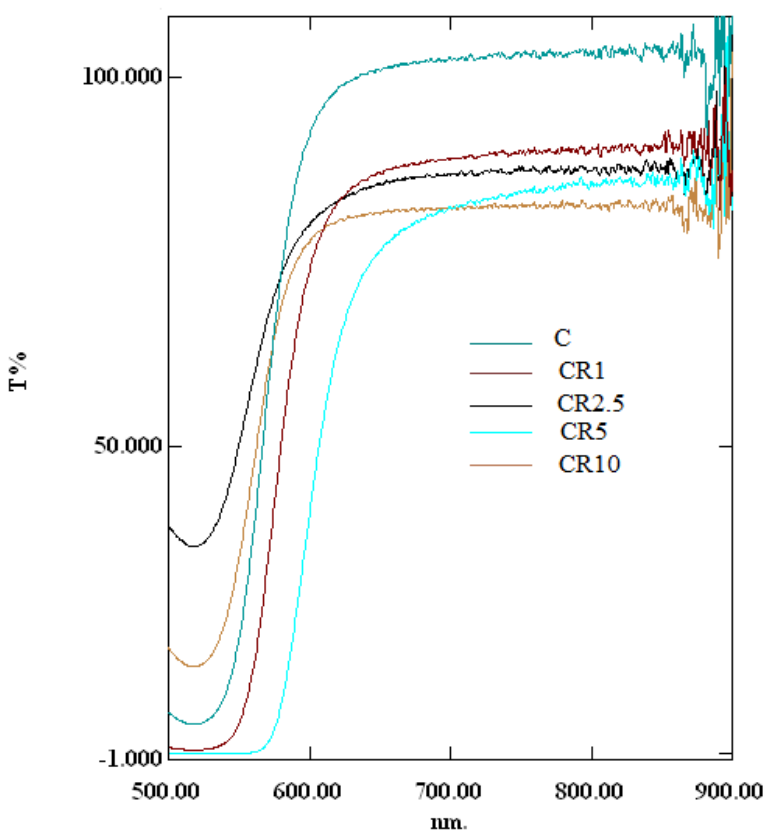

Figure 3: UV transmittance of all biobased films 
The color values of the biobased films in acidic condition and alkaline condition are given in Table 2. Color differences were calculated by delta $\mathrm{E}$ of films (C, CR1, CR2.5, CR5 and CR10) how it differs from acidic and alkaline conditions. When the table is examined, it is seen that the colors of the biobased films are brown-red in acidic conditions. However, when the films are brought into contact with the base, the colors suddenly turn green. The change in colors can be perceived by the eye, as well as evidenced by spectral measurements. When the color differences in acidic and alkaline conditions were examined, it was determined that the difference was 7.93 even in the formulation containing the least rosehip extract. As the amount of rosehip extract increases, the color difference increases rapidly. However, as the amount of rosehip extract increases, areas of different color intensity (heterogeneous colored regions) may occur on the film. It can be planned to be used in smart packaging applications because of the color change feature of rosehip at different $\mathrm{pH}$ and color change will occur due to bases such as ammonia released from spoiled foods. When the gloss value was examined, it was measured that the gloss of all biobased films was close to each other. However, as the amount of rosehip increases, there is a small decrease in gloss. This is due to the roughness of the surface.

Table 2: Color and gloss properties of biobased films

\begin{tabular}{|c|c|c|c|c|c|c|c|}
\hline Formulation & $\mathrm{L}^{*}$ & $a^{*}$ & b* & Image (acid) & $\begin{array}{l}\text { Image } \\
\text { (base) }\end{array}$ & $\Delta \mathrm{E}_{00}$ & Gloss \\
\hline C & 83 & -1 & 2 & & & 1.62 & 28 \\
\hline CR1 & 58 & 12 & 43 & & & 7.93 & 26 \\
\hline CR2.5 & 44 & 14 & 41 & 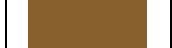 & & 15.02 & 26 \\
\hline CR5 & 26 & 20 & 32 & & & 23.72 & 25 \\
\hline CR10 & 23 & 21 & 28 & & & 25.09 & 24 \\
\hline
\end{tabular}

The surface energies and water contact angles (WCA) of the biobased films are presented in Table 3. The WCA of base formulation film (C) was found as $72.7^{\circ}$. When rosehip extract was added to $C$ film, the contact angle increased to $76.7^{\circ}$. As the amount of rosehip in the formulation increases, the contact angle increases slightly. The reason for this is that the rosehip creates some indentation on the surface and therefore the lotus effect occurs.

Table 3. Total surface energy and contact angle values of the all biobased films

\begin{tabular}{|c|c|c|}
\hline Formulation & $\begin{array}{c}\text { Surface Energy } \\
\left(\mathbf{m J} / \mathbf{m}^{\mathbf{2}}\right)\end{array}$ & $\begin{array}{c}\text { Contact Angle } \\
\left.\mathbf{(}^{\circ}\right)\end{array}$ \\
\hline C & 38.7 & 72.7 \\
\hline CR1 & 37.3 & 76.7 \\
\hline CR2.5 & 37.1 & 76.9 \\
\hline CR5 & 36.9 & 77.1 \\
\hline CR10 & 36.7 & 77.4 \\
\hline
\end{tabular}




\section{CONCLUSIONS}

When the data obtained were examined, it was concluded that the film containing rosehip could be used in active packaging. When the color results are examined, it is seen that the color is brown in acidic conditions and green in basic conditions, and the color difference varies between 7-25. This shows that the color change can be easily detected both with the eye and with the spectrophotometer. Thus, an inexpensive, easily detectable, effective active packaging material was produced. As the amount of rosehip added to the medium increases, the amount of active substance added should be kept under control, since heterogeneous color distribution is obtained. The gloss values of the film obtained are quite high. However, as the amount of rosehip increased, there was a slight decrease in gloss. Likewise, the contact angle increased slightly with the lotus effect caused by surface roughness. However, this increase can be ignored.

\section{REFERENCES}

[1] Aimin Huang, A., Wei, L., Zhao, Z., Wei, G., Zhang, Y., Huang, Z., Li, X., Hu, H., Qin, X., Yang, M.: “A comparative analysis of the preparation of cellulose acetate butyrate and the characteristics of applying in pearlescent coating film", Polymer Bulletin, 77, 2873-2887, 2020. doi: 10.1007/s00289019-02886-w.

[2] Çakmakçı, E., Güngör, A.: "Preparation and characterization of flame retardant and proton conducting boron phosphate/polyimide composites", Polymer Degradation and Stability 98 (5), 927-933, 2013. doi: 10.1016/j.polymdegradstab.2013.03.003.

[3] Edgar K. J.: "Cellulose esters in drug delivery", Cellulose, 14, 49 -64, 2007. doi: 10.1007/s10570006-9087-7.

[4] Furtado, L. M., Hilamatu, K. C. P., Balaji, K., Ando, R. A., Petri, D. F. S.: "Miscibility and sustained release of drug from cellulose acetate butyrate/caffeine films", Journal of Drug Delivery Science and Technology 55, 101472-101479, 2020. doi: 10.1016/j.jddst.2019.101472.

[5] Ghasemi-Varnamkhasti, M., Mohammad-Razdari, Ayat., Yoosefian S. H., Izadi, Z.: "Effects of the combination of gamma irradiation and $\mathrm{Ag}$ nanoparticles polyethylene films on the quality of fresh bottom mushroom (Agaricus bisporus L.)", Journal of Food Processing and Preservation 42 (7), 13652-13659, 2018. doi: 10.1111/jfpp.13652.

[6] Go, E. J., Song, K. B.: "Antioxidant properties of rye starch films containing rosehip extract and their application in packaging of chicken breast" Starch-Stärke 71 (11-12), 1900116, 2019. doi: 10.1002/star.201900116.

[7] Idumah, C. I., Zurina, M., Ogbu, J., Ndem, J. U., Igba, E. C.: "A review on innovations in polymeric nanocomposite packaging materials and electrical sensors for food and agriculture", Composite Interfaces 27 (1), 1-72, 2020. doi: 10.1080/09276440.2019.1600972.

[8] Liu, Y., Qin, Y., Bai, R., Zhang, X., Yuan, L., Liu, J.: "Preparation of pH-sensitive and antioxidant packaging films based on k-carrageenan and mulberry polyphenolic extract", International Journal of Biological Macromolecules 134, 993-1001, 2019. doi: 10.1016/j.ijbiomac.2019.05.175.

[9] Saha, N. R., Roy, I., Sarkar, G., Bhattacharyya, A., Das, R., Rana, D., Banerjee, R., Pau, A. K., Mishra, R., Chattopadhyay, D.: "Development of active packaging material based on cellulose acetate butyrate/polyethylene glycol/aryl ammonium cation modified clay", Carbohydrate Polymers 187, 818, 2018. doi: 10.1016/j.carbpol.2018.01.065.

[10] Shokri, J., Adibkia, K.: "Application of Cellulose and Cellulose Derivatives in Pharmaceutical Industries", In: Cellulose - Medical, Pharmaceutical and Electronic Applications, (IntechOpen Limited, London, 2013). doi: 10.5772/55178.

[11] Sobral, M. C. C. M., Sobral, A. J. F. N., Guthrie, J. T., Gil, M. H.: "Ketotifen controlled release from cellulose acetate propionate and cellulose acetate butyrate membranes", Journal of Materials Science: Materials in Medicine 19,677-682, 2008. doi: 10.1007/s10856-007-0168-4.

[12] Zhang, H., Hou, A., Xie, K., Gao, A.: "Smart color-changing paper packaging sensors with pH sensitive chromophores based on azo-anthraquinone reactive dyes", Sensors and Actuators B: Chemical 286, 362-369, 2019. doi: 10.1016/j.snb.2019.01.165. 


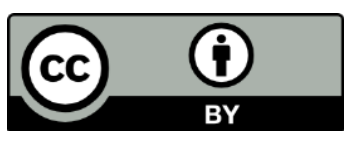

(C) 2020 Authors. Published by the University of Novi Sad, Faculty of Technical Sciences, Department of Graphic Engineering and Design. This article is an open access article distributed under the terms and conditions of the Creative Commons Attribution license 3.0 Serbia (http://creativecommons.org/licenses/by/3.0/rs/). 